\title{
Impact Assessment of Horticulture Fair on Farming Community
}

\author{
B.S. Lakshmana Reddy ${ }^{1}$, Pushpa ${ }^{1}$ and M.V. Srinivas Reddy ${ }^{2}$ \\ ${ }^{1}$ Agricultural Extension, Department of Social and Allied Sciences, University of Horticultural \\ Sciences Bagalkot, Karnataka, India \\ ${ }^{2}$ Agricultural Extension, UAS, Bengaluru, Karnataka, India \\ *Corresponding author
}

\begin{abstract}
A B S T R A C T
Horticulture fair is an integrated extension approach to disseminate the technologies to its stakeholders and provides an opportunity to know about newly released technologies and ongoing research in the field. The present study was conducted during Totagarike Mela of University of Horticultural Sciences, Bagalkot. The data was collected from 500 participants using structured schedule. The results reveal that majority of the respondents are farmers $(94.20 \%)$ belong to Bagalkot district $(49.40 \%)$, participated only once $(59.20 \%)$, illiterates $(39.20 \%)$ and aged between 30 to 50 years $(52.80 \%)$. The respondents obtained information about mela from Daily Newspaper (27.00\%), Development Departments and Television $(21.40 \%$ each) and Friends $(20.40 \%)$.They participated to get technical information(63.80\%) and to see indoor exhibition (28\%) and implements and machineries $(25.40 \%)$ while only $34.20 \%$ of them viewed all the stalls. About 47.40 per cent of respondents visited indoor exhibition followed by agricultural machineries $(45.60 \%)$, soil world $(44.20 \%)$ and horticulture $(38.20 \%)$. About 45.60per cent of the respondents received new information about agricultural machineries followed by soil world (32.80\%), indoor exhibition (26.40\%) and horticulture (22.60\%) while only 56.20per cent of them visited demonstration plots and 28.60per cent participated in farmer to farmer interaction. Among 158 respondents who received useful and innovative technology/information, only 56.32per cent said they will adopt. Only 47.20per cent of participant's said they will motivate others to participate in the Mela.
\end{abstract}

\section{Keywords}

Horticulture, Farming community, Agricultural machineries

\section{Article Info}

Accepted:

15 July 2020 Available Online: 10 August 2020

\section{Introduction}

Horticulture fair is an important event which plays a pivotal role in transfer of technologies to the stakeholders of agriculture, horticulture and related enterprises. It envisages a large scale display of improved horticultural technologies and farm inputs for the benefit of farmers at one roof.it is an integrated extension approach consisting of all the extension teaching methods Viz. individual, group as well as mass contact methods with appropriate audio visual aids. Owing to its powerfulness in changing the behaviour of farmers, this mega event is organized annually involving other line departments and 
institutions to transfer the technologies to the intended clientele.

University of Horticultural Sciences (UHS), Bagalkot organized its fourth Totagarika Mela from $19-21^{\text {st }}$ December, 2015 on a theme "Healthy Soils for a Healthy Life" providing a forum for interaction and throws light on development and opportunities in horticulture and allied sectors in the domestic and international arena especially on soil health, post harvest technology, seed processing, soil and water conservation practices, organic farming, green house technology and farm machinery. The main purpose of organizing Totagarikemela was to provide first hand information to farmers about the availability of modern technology useful to stakeholders and also to update about current ongoing research activities on various problems of farming. Nearly three lakh farmers from all the corners of the state and few from neighbouring state were participated. Around 450 horti-based companies and institutions participated in the mela to reach the end users. It is a great opportunity for the agripreneurs, developmental departments and nongovernmental organizations and financial institutions to take part in this event.

At present, there has been increasing demand for organizing this type of Krishimelas and horti-fairs at various levels. with this background the present study was conducted with the following specific objectives to study the socio-personal characteristics of farmers participated in Totagarika Mela, to know the source of information to participate in mela, to understand the perception of farmers about usefulness of mela, to identify and designate the purpose for their participation and to document the suggestions of the farmers for the improvement for the conduct of melas in future. The findings on these aspects would help as to identify the strength and weakness of the event and also to tap the area that needs toning up and fine tuning preparations.

\section{Materials and Methods}

The present study was conducted during Totagarike Mela of University of Horticultural Sciences, Bagalkot organized for three days from 19th to $21^{\text {st }}$ December, 2015. About 500 participants of Totagarike Mela were randomly contacted with the help of structured schedule for eliciting the information by personal interview method. The data was analyzed using frequency and percentage.

\section{Results and Discussion}

The findings of the present study have been summarized as below:

\section{District wise participation of respondents}

Table 1 depicts that nearly half of the participant respondents were come from the Bagalkot (49.40\%) followed by Vijayapura (12.80\%), Belgaum (7\%), Bellary (6\%), Dharwad (4\%), Koppal (3.40\%), Bidar $(2.80 \%)$ and Chitradurga $(2.0 \%)$. The participants from remaining districts namely UK, Karwar, Yadagiri, Raichur, Gadag, Haveri, Davanagere, Chikkaballpur, Shivamoga, Kalburgi, Bengaluru urban, Bengaluru rural, Mysuru and Kolar were less than two percent in each of these districts.

\section{Frequency of participation of respondents}

From the Table 2, we can conclude that around 60 per cent of the respondent farmers participated for the first time in the Totakarike Mela while 24 per cent of the respondents participated for the second time. Only 10 per cent of the respondents participated from three years but 6.80 per cent of the respondents participated in all the four Totagarike Mela's organized by UHS, Bagalkot. 


\section{Age of the respondents}

From the table 3, we can opine that 27per cent of the respondents belong to age group of 41 to 50 years followed by 31 to 40 years age (25.80).

While, 17.20 per cent of the respondents were having age between 21 to 30years and 15.20 and 12.80 per cent of the respondents belong to age group of 51-60 \& more than 60 years respectively. Only two per cent of respondents were below 20years of age.

\section{Education}

From the Table 4, we can depict the categorization of respondents based on education level, nearly 40 percent of the respondents were illiterates followed by high school $(15.60 \%)$, graduate $(14.40 \%)$, preuniversity $(13.00 \%)$ and higher primary $(10.00 \%)$. While post graduates and primary school respondents were 5.20 and 2.60 per cent respectively.

\section{Land holdings of the participants}

Table 5 depicts that nearly $1 / 4^{\text {th }}$ of the respondents were having 3to 4acres of land while 21per cent of them have 5 to 6 acres. Around 17.20per cent respondents have 7 to 8 acres, 14per cent have 1to 2 acres, 12.60per cent have 9 to 10 acres and 10.80per cent have 11 acres and above.

\section{Occupation of the respondents}

Table 6 depicts that majority of the respondents are agriculturists $(94.20 \%)$ while only 1.80per cent respondents were businessmen and 4 per cent of them were employed in other jobs viz, public and private sector.

\section{Subsidiary activities of the respondents}

The analysis of subsidiary activities of the respondents reveals that 6.60per cent of the respondents practice agriculture as a subsidiary activity followed by dairy $(4.80 \%)$ and business $(1.40 \%)$. Less than one percent were working as a labour, practicing poultry, sheep rearing, consultancy, horticulture, piggery, tailor and lawyer (Table 7).

\section{Type of cultivation}

With regard to cultivation, 56.20per cent of the respondents are having irrigation facility while remaining 43.80per cent were practicing dryland farming (Table 8).

\section{Source of information about Mela}

With regard to source of information (Table 9 ), we can opine that just more than $1 / 4^{\text {th }}$ of the respondents got information about Mela from daily newspaper (27.00\%) followed by television and development departments (21.40 each) and friends (20.40\%). While very less number of respondents got information from radio $(4.80 \%)$, NGOs $(3.60 \%)$ and only one percent got information from social media. About four per cent told that they got information other than the above said sources.

\section{Facilitating Agency for the transportation of the respondents}

Nearly 60 per cent of the respondents participated through their own means while development departments and University arranged the transportation for about 18.60per cent and 13.60per cent respondents respectively (Table 10). NGOs facilitated transportation for about 4.80per cent of the respondents while 2.60per cent respondents got transportation through friends, trusts, etc. 


\section{Purpose of participation}

With regard to the purpose of participation, more than half of the respondents participated to get technical information $(63.80 \%)$ followed by 28per cent participation to see indoor exhibition, 25.40per cent participated to see the implements and machineries while 21.20per cent participated to see demonstration plots, 18per cent discussed with the scientist and 10.20per cent purchased the agricultural implements. About 4.80 participated without any of the purposes mentioned above while 12.60per cent of the respondents participated for other purposes may be their friend, neighbours, colleagues might brought forcefully or for curiosity or to meet friends, etc. (Table 11).

\section{Type of exhibits viewed by the respondents}

Table 12 depicts that only 34.20per cent respondent were able to view all the stalls while remaining have seen few important stalls necessary for them. About 47.40per cent of respondents visited indoor exhibition followed by agricultural machineries $(45.60 \%)$, soil world $(44.20 \%)$, horticulture (38.20\%), livestock $(28.80 \%)$ and University stalls $(24.40 \%)$. Less than $1 / 4^{\text {th }}$ of the total respondents visited other stalls like development departments (17.60\%), private companies (15\%), research stations $(12.80 \%)$, publication, consultancy cell and Agricultural implements (11.00\% each). About 24.80per cent of the respondents visited food stalls and other refreshments.

\section{Respondents received new information from various stalls}

The data reveals that no respondent received information from all the stalls in the Mela because there were too many stalls and cannot be exposed in a single day. About 45.60per cent of the respondents received new information about agricultural machineries followed by soil world (32.80\%), indoor exhibition $(26.40 \%)$, horticulture $(22.60 \%)$, research demonstration (14.60\%), livestock exhibition (12.80\%) and food stalls (10.80\%). Less than $1 / 10^{\text {th }}$ of the respondents received new information from consultancy of scientists $(9.20 \%)$ followed by private companies $(7.60 \%)$, agricultural implements (7.40\%), publication (5.80\%), University stalls $(5.00 \%)$, research stations $(3.40 \%)$, development departments (3.20\%) and field demonstrations $(1.80 \%)$ (Table 13).

\section{Inputs / Implements purchased by the respondents}

From this table 14, we can conclude that only 10per cent of the respondents purchased seeds followed by seedlings $(8.20 \%)$, implements $(6.80 \%)$, publications $(5.00 \%)$ and fertilizers (4.40\%). But 18.60per cent of the respondents purchased other inputs like biofertilizers, insecticides or pesticides, etc.

\section{Participants visited demonstration plots}

Table 15 denotes that about 56.20per cent of the respondents were visited demonstration plots whereas remaining 43.80per cent of them didn't visited demonstration plots may be due to lack of time, lack of awareness, etc.

Demonstration plots visited by the participants

Table 16 data reveals that in fruit science division, majority of the respondents (58.20\%) visited mango demonstration plots followed by pomegranate (26\%), grapes $(22.20 \%)$, Guava $(16.80 \%)$, sapota $(19.80 \%)$, custard apple (12.80\%), citrus (8.20\%), Amla $(1.80 \%)$, Mosambi (1.60\%), jack fruit $(1.00 \%)$ and other plots (1.40\%). In vegetable division, majority of the respondents visited drumstick $(51.60 \%)$, onion $(23.80 \%)$, field 
bean $(17.80 \%)$, pumpkin $(8.40 \%)$ and others $(2.80 \%)$. In floriculture division, majority of the respondents visited rose garden $(57.0 \%)$ followed by Jasmine (16.80\%), Gerbera $(20.60 \%)$ and other flowers gardens $(1.0 \%)$. In plantation, spices, medicinal and ornamental division, majority of the respondents visited coconut (39.60\%) and only one per cent of the respondents visited other crops. Very few respondents visited agro forestry $(1.80 \%)$, IFS unit $(2.60 \%)$ and other divisions $(0.80 \%)$

\section{Farmer's most interested division/part of the Mela}

Table 17 depicts that nearly half of the respondents $(49.80 \%)$ expressed their most interested division/part as indoor exhibition followed by consultancy cell (16.20\%), demonstrations $(11.60 \%)$, farmer to farmer interaction (11.60\%), field visits $(9.20 \%)$, stalls (8.60\%), farmer award function $(6.80 \%)$, input availability $(5.80 \%)$ and other divisions $(1.80 \%)$.

\section{Three best stalls as perceived by the respondents}

The responses of the respondents about their perception of three best stalls of totagarike mela were depicted in table 18. About 18.60per cent of the respondents have perceived floriculture as the best division/ stall followed by agriculture implements/ machineries (17.20\%), vegetable division $(16.40 \%)$ and fruit division (16.20\%). Less than $1 / 10^{\text {th }}$ of the farmers perceived Soil world (9.20\%), Consultancy (6.80\%), Livestock (6.20\%), Out-door Exhibition $(5.40 \%)$, Indoor exhibition (3.20\%), Seed division (2.40\%), Entomology (2.00\%) and $1.60 \%$ of Dog show, Solar based machineries, Food stalls, Private institutes and NGO. About one per cent of the respondents perceived Post Harvest technology division as the best followed by $0.80 \%$ each of the Medicinal and aromatic division, Social and allied sciences and Banana stalls. About $0.60 \%$ each of the respondents perceived Plant pathology division, Publication, Organic farming, KVK, Kolar and Rabbit rearing while only one person each perceived Research division, Natural Resource Management, Biofuel, Sheep \& goat rearing, UAHS, Shivmogga and UAS, Bengaluru as the best division/stalls

\section{Participation in farmer-farmer Interaction}

Out of the total 500 respondents, only 143 respondents $(28.60 \%)$ participated in farmer to farmer interaction while remaining 357 respondents $(71.40 \%)$ didn't participate in farmer to farmer interaction (Table 19).

\section{Respondents opinion about farmer-farmer Interaction}

Out of the 143 respondents participated in the farmer to farmer interaction programmes, $39.16 \%$ of them expressed it is best while 41.96per cent told better followed by 18.18 per cent as useful. Only one $(0.70 \%)$ respondents opined useless about farmerfarmer Interaction (Table 20).

\section{Opinion about the facilities provided to the respondents}

The opinion of the respondents about the facilities provided to them is mentioned as below. With regard to the transportation facility, majority of them opined best $(51.80 \%)$ followed by better (37.20\%), normal (7.80\%) and poor (3.20\%).With respect to interaction with scientists, $44.60 \%$ of them expressed better followed by best (41.80\%), normal (11.40\%) and poor $(2.00 \%)$.With regard to the publicity about the Mela, nearly half of them $(50.20 \%)$ opined best followed by better $(41.40 \%)$, normal 
$(6.60 \%)$ and poor $(1.80 \%)$.The food arrangement was better as expressed by $48.0 \%$ respondents followed by best $(29.0 \%)$, normal $(18.6 \%)$ and poor $(4.40 \%)$.

With respect to drinking water facility, majority of the respondents (49.20\%) expressed better followed by best $(31.60 \%)$, normal (14.40\%) and poor (4.80\%).The parking facility provided was best as opined by $46 \%$ respondents followed by better $(45.80 \%)$, normal $(7.0 \%)$ and poor $(1.20 \%)$. Majority of them said that the toilet facility was better $(53.20 \%)$ followed by best $(32 \%)$, Normal $(13 \%)$ and poor $(1.80 \%)$. More than half of the respondents $(51.20 \%)$ expressed that accommodation was better followed by best $(23.80 \%)$, normal $(19.80 \%)$ and poor $(5.20 \%)$ (Table 21).

Useful and innovative technology/ information as perceived by the respondents

Table 22 depicts that around 13.60per cent of the respondents perceived that technical information is very useful followed by machineries and implements $(9.20 \%)$, solar machineries $(5.00 \%)$, demonstrations $(3.60 \%)$ and livestock $(0.2 \%)$.

Table.1 District wise participation of respondents

\begin{tabular}{|c|c|c|c|}
\hline Sl. No. & District & Number & Percent \\
\hline 1. & Bagalkot & 247 & 49.40 \\
\hline 2. & Vijayapur & 64 & 12.80 \\
\hline 3. & Belgaum & 35 & 7.00 \\
\hline 4. & Bellary & 30 & 6.00 \\
\hline 5. & Dharwad & 20 & 4.00 \\
\hline 6. & Koppal & 17 & 3.40 \\
\hline 7. & Bidar & 14 & 2.80 \\
\hline 8. & Chitradurga & 10 & 2.00 \\
\hline 9. & Uttar kannada & 9 & 1.80 \\
\hline 10. & Karwar & 9 & 1.80 \\
\hline 11. & Yadagiri & 7 & 1.40 \\
\hline 12. & Raichur & 7 & 1.40 \\
\hline 13. & Gadag & 7 & 1.40 \\
\hline 14. & Haveri & 5 & 1.00 \\
\hline 15. & Davanagere & 4 & 0.80 \\
\hline 16. & Chikkaballapur & 3 & 0.60 \\
\hline 17. & Shivamoga & 3 & 0.60 \\
\hline 18. & Kalburgi & 3 & 0.60 \\
\hline 19. & Bengaluru Urban & 2 & 0.40 \\
\hline 20. & Bengaluru rural & 1 & 0.20 \\
\hline 21. & Mysuru & 1 & 0.20 \\
\hline \multirow[t]{2}{*}{22.} & Kolar & 2 & 0.40 \\
\hline & Total & 500 & 100.00 \\
\hline
\end{tabular}


Table.2 Distribution of respondents based on their frequency of participation

\begin{tabular}{|c|l|c|c|}
\hline Sl. No. & Frequency & Number & Per cent \\
\hline 1. & Once & 296 & 59.20 \\
\hline $\mathbf{2 .}$ & Twice & 120 & 24.00 \\
\hline 3. & Thrice & 50 & 10.00 \\
\hline $\mathbf{4 .}$ & Four times & 34 & 6.80 \\
\hline & Total & $\mathbf{5 0 0}$ & 100.00 \\
\hline
\end{tabular}

Table.3 Distribution of respondents based on age

\begin{tabular}{|c|c|c|c|}
\hline Sl. No. & Age & Number & Per cent \\
\hline $\mathbf{1 .}$ & $<20$ & 10 & 2.00 \\
\hline $\mathbf{2 .}$ & $21-30$ & 86 & 17.20 \\
\hline $\mathbf{3 .}$ & $31-40$ & 129 & 25.80 \\
\hline $\mathbf{4 .}$ & $41-50$ & 135 & 27.00 \\
\hline $\mathbf{5 .}$ & $51-60$ & 76 & 15.20 \\
\hline $\mathbf{6 .}$ & $>60$ & 64 & 12.80 \\
\hline & Total & $\mathbf{5 0 0}$ & $\mathbf{1 0 0 . 0 0}$ \\
\hline
\end{tabular}

Table.4 Categorization of the respondents based on Education

\begin{tabular}{|c|l|c|r|}
\hline $\begin{array}{c}\text { Sl. } \\
\text { No. }\end{array}$ & Education level & Number & Per cent \\
\hline 1. & Illiterate & 196 & 39.20 \\
\hline 2. & Primary school & 13 & 2.60 \\
\hline 3. & Higher primary & 50 & 10.00 \\
\hline $\mathbf{4 .}$ & High school & 78 & 15.60 \\
\hline $\mathbf{5 .}$ & Pre University & 65 & 13.00 \\
\hline 6. & Graduate & 72 & 14.40 \\
\hline $\mathbf{7 .}$ & Post graduate & 26 & 5.20 \\
\hline & Total & $\mathbf{5 0 0}$ & 100.00 \\
\hline
\end{tabular}

Table.5 Distribution of respondents based on landholdings

\begin{tabular}{|c|c|c|c|}
\hline Sl. No. & Land holdings (acres) & Number & Per cent \\
\hline $\mathbf{1 .}$ & $1-2$ & 70 & 14.00 \\
\hline $\mathbf{2 .}$ & $3-4$ & 122 & 24.40 \\
\hline $\mathbf{3 .}$ & $5-6$ & 105 & 21.00 \\
\hline $\mathbf{4 .}$ & $7-8$ & 86 & 17.20 \\
\hline $\mathbf{5 .}$ & $9-10$ & 63 & 12.60 \\
\hline $\mathbf{6 .}$ & 11 and above & 54 & 10.80 \\
\hline & Total & 500 & 100.00 \\
\hline
\end{tabular}


Table.6 Main occupation of the respondents

\begin{tabular}{|l|c|c|c|}
\hline Sl. No. & Occupation & Number & Per cent \\
\hline $\mathbf{1 .}$ & Agriculture & 471 & 94.20 \\
\hline $\mathbf{2 .}$ & Business & 9 & 1.80 \\
\hline $\mathbf{3 .}$ & Job & 20 & 4.00 \\
\hline & Total & $\mathbf{5 0 0}$ & $\mathbf{1 0 0 . 0 0}$ \\
\hline
\end{tabular}

Table.7 Subsidiary activities of the respondents

\begin{tabular}{|c|l|c|c|}
\hline Sl. No. & \multicolumn{1}{|c|}{ Activity } & Number & Per cent \\
\hline $\mathbf{1 .}$ & Agriculture & 33 & 6.60 \\
\hline $\mathbf{2 .}$ & Dairy & 24 & 4.80 \\
\hline $\mathbf{3 .}$ & Business & 7 & 1.40 \\
\hline $\mathbf{4 .}$ & Labour & 4 & 0.80 \\
\hline $\mathbf{5 .}$ & Poultry & 4 & 0.80 \\
\hline $\mathbf{6 .}$ & Sheep rearing & 3 & 0.60 \\
\hline $\mathbf{7 .}$ & Consultancy & 3 & 0.60 \\
\hline $\mathbf{8 .}$ & Horticulture & 1 & 0.20 \\
\hline $\mathbf{9 .}$ & Piggery & 1 & 0.20 \\
\hline $\mathbf{1 0 .}$ & Tailor & 1 & 0.20 \\
\hline $\mathbf{1 1 .}$ & Lawyer & 1 & 0.20 \\
\hline
\end{tabular}

Table.8 Type of cultivation

\begin{tabular}{|c|l|c|c|}
\hline SI. No. & Type of cultivation & Number & Per cent \\
\hline 1. & Irrigated & 281 & 56.20 \\
\hline 2. & Dry land & 219 & 43.80 \\
\hline
\end{tabular}

Table.9 Source of information about Mela

\begin{tabular}{|c|l|c|c|}
\hline Sl. No. & Source of information & Number & Per cent \\
\hline $\mathbf{1 .}$ & Daily Newspaper & 135 & 27.00 \\
\hline $\mathbf{2 .}$ & Television & 107 & 21.40 \\
\hline $\mathbf{3 .}$ & Development Departments & 107 & 21.40 \\
\hline $\mathbf{4 .}$ & Friends & 102 & 20.40 \\
\hline $\mathbf{5 .}$ & UHS & 94 & 18.80 \\
\hline $\mathbf{6 .}$ & Radio & 24 & 4.80 \\
\hline $\mathbf{7 .}$ & NGOs & 18 & 3.60 \\
\hline $\mathbf{8 .}$ & Social Media & 5 & 1.00 \\
\hline $\mathbf{9 .}$ & Others & 20 & 4.00 \\
\hline
\end{tabular}


Table.10 Facilitating agency for the transportation of the respondents

\begin{tabular}{|c|l|c|c|}
\hline Sl. No. & \multicolumn{1}{|c|}{ Agency } & Number & Per cent \\
\hline $\mathbf{1 .}$ & Own means & 302 & 60.40 \\
\hline $\mathbf{2 .}$ & Development departments & 93 & 18.60 \\
\hline $\mathbf{3 .}$ & University & 68 & 13.60 \\
\hline $\mathbf{4 .}$ & NGOs & 24 & 4.80 \\
\hline $\mathbf{5 .}$ & Others & 13 & 2.60 \\
\hline
\end{tabular}

Table.11 Purpose of participation

\begin{tabular}{|l|l|c|c|}
\hline $\begin{array}{c}\text { Sl. } \\
\text { No. }\end{array}$ & \multicolumn{1}{|c|}{ Purpose } & Number & Per cent \\
\hline 1. & To get Technical information & 319 & 63.80 \\
\hline 2. & To see Indoor exhibition & 140 & 28.00 \\
\hline 3. & To see implements and machineries & 127 & 25.40 \\
\hline 4. & To see demonstration plots & 106 & 21.20 \\
\hline $\mathbf{5 .}$ & To discuss with scientists & 90 & 18.00 \\
\hline $\mathbf{6 .}$ & To purchase agricultural implements & 51 & 10.20 \\
\hline 7. & None of the above & 24 & 4.80 \\
\hline $\mathbf{8 .}$ & Other & 63 & 12.60 \\
\hline
\end{tabular}

Table.12 Type of exhibits viewed by the respondents

\begin{tabular}{|l|l|c|c|}
\hline Sl. No. & \multicolumn{1}{|c|}{ Exhibitions / stalls } & Number & Per cent \\
\hline $\mathbf{1 .}$ & Indoor exhibitions & 237 & 47.40 \\
\hline $\mathbf{2 .}$ & Agricultural Machineries & 228 & 45.60 \\
\hline $\mathbf{3 .}$ & Soil world & 221 & 44.20 \\
\hline $\mathbf{4 .}$ & Horticulture & 191 & 38.20 \\
\hline $\mathbf{5 .}$ & Livestock & 144 & 28.80 \\
\hline $\mathbf{6 .}$ & Research demonstrations & 143 & 28.60 \\
\hline $\mathbf{7 .}$ & University stalls & 122 & 24.40 \\
\hline $\mathbf{8 .}$ & Development departments & 88 & 17.60 \\
\hline $\mathbf{9 .}$ & Private companies & 75 & 15.00 \\
\hline $\mathbf{1 0 .}$ & Research stations & 64 & 12.80 \\
\hline $\mathbf{1 1 .}$ & Publication & 55 & 11.00 \\
\hline $\mathbf{1 2 .}$ & Consultancy cell & 55 & 11.00 \\
\hline $\mathbf{1 3 .}$ & Agricultural implements & 55 & 11.00 \\
\hline $\mathbf{1 4 .}$ & Field demonstrations & 38 & 7.60 \\
\hline $\mathbf{1 5 .}$ & Food stalls & 124 & 24.80 \\
\hline $\mathbf{1 6 .}$ & All stalls & 171 & 34.20 \\
\hline
\end{tabular}


Table.13 Number of respondents received new information from various stalls

\begin{tabular}{|c|l|c|c|}
\hline SI. No. & \multicolumn{1}{|c|}{ Exhibitions / stalls } & Number & Per cent \\
\hline 1. & Agricultural Machineries & 228 & 45.60 \\
\hline $\mathbf{2 .}$ & Soil world & 164 & 32.80 \\
\hline $\mathbf{3 .}$ & Indoor exhibitions & 132 & 26.40 \\
\hline $\mathbf{4 .}$ & Horticulture & 113 & 22.60 \\
\hline $\mathbf{5 .}$ & Research demonstrations & 73 & 14.60 \\
\hline $\mathbf{6 .}$ & Livestock exhibition & 64 & 12.80 \\
\hline $\mathbf{7 .}$ & Food stalls & 54 & 10.80 \\
\hline $\mathbf{8 .}$ & Consultancy cell & 46 & 9.20 \\
\hline $\mathbf{9 .}$ & Private companies & 38 & 7.60 \\
\hline $\mathbf{1 0 .}$ & Agricultural implements & 37 & 7.40 \\
\hline $\mathbf{1 1 .}$ & Publication & 29 & 5.80 \\
\hline $\mathbf{1 2 .}$ & University stalls & 25 & 5.00 \\
\hline $\mathbf{1 3 .}$ & Research stations & 17 & 3.40 \\
\hline $\mathbf{1 4}$ & Development departments & 16 & 3.20 \\
\hline $\mathbf{1 5 .}$ & Field demonstrations & 9 & 1.80 \\
\hline $\mathbf{1 6 .}$ & All stalls & 0 & 0.00 \\
\hline
\end{tabular}

Table.14 Inputs / Implements purchased by the respondents

\begin{tabular}{|c|l|c|c|}
\hline Sl. No. & Inputs / Implements & Number & Per cent \\
\hline 1. & Seeds & 50 & 10.00 \\
\hline 2. & Seedlings & 41 & 8.20 \\
\hline 3. & Implements & 34 & 6.80 \\
\hline $\mathbf{4 .}$ & Publications & 25 & 5.00 \\
\hline $\mathbf{5 .}$ & Fertilizers & 22 & 4.40 \\
\hline $\mathbf{6 .}$ & Other inputs & 93 & 18.60 \\
\hline
\end{tabular}

Table.15 Number of participants visited demonstration plots

\begin{tabular}{|c|l|c|c|}
\hline SI. No. & Particulars & Number & Per cent \\
\hline 1. & Visited & 281 & 56.20 \\
\hline 2. & Not visited & 219 & 43.80 \\
\hline
\end{tabular}


Table.16 Details of demonstration plots visited by the participants

\begin{tabular}{|c|c|c|c|c|}
\hline Sl. No. & Division & Crop & Number & Per cent \\
\hline \multirow[t]{11}{*}{1.} & \multirow[t]{11}{*}{ Fruit science } & Mango & 291 & 58.20 \\
\hline & & Pomegranate & 130 & 26.00 \\
\hline & & Grapes & 111 & 22.20 \\
\hline & & Guava & 84 & 16.80 \\
\hline & & Sapota & 99 & 19.80 \\
\hline & & Custard apple & 64 & 12.80 \\
\hline & & Citrus & 41 & 8.20 \\
\hline & & Amla & 9 & 1.80 \\
\hline & & Mosambi & 8 & 1.60 \\
\hline & & Jack fruit & 5 & 1.00 \\
\hline & & Others & 7 & 1.40 \\
\hline \multirow[t]{5}{*}{2.} & \multirow[t]{5}{*}{ Vegetable } & Drumstick & 258 & 51.60 \\
\hline & & Onion & 119 & 23.80 \\
\hline & & Field bean & 89 & 17.80 \\
\hline & & Pumpkin & 42 & 8.40 \\
\hline & & Others & 14 & 2.80 \\
\hline \multirow[t]{4}{*}{3.} & \multirow[t]{4}{*}{ Floriculture } & Rose & 285 & 57.00 \\
\hline & & Jasmine & 84 & 16.80 \\
\hline & & Gerbera & 103 & 20.60 \\
\hline & & Others & 5 & 1.00 \\
\hline \multirow[t]{2}{*}{4.} & \multirow{2}{*}{$\begin{array}{l}\text { Plantation, spices, medicinal } \\
\text { and ornamental }\end{array}$} & Coconut & 198 & 39.60 \\
\hline & & Others & 5 & 1.00 \\
\hline 5. & Agro forestry & & 9 & 1.80 \\
\hline 6. & IFS unit & & 13 & 2.60 \\
\hline 7. & Other divisions & & 4 & 0.80 \\
\hline
\end{tabular}

Table.17 Farmer's most interested division/part of the Mela

\begin{tabular}{|c|l|c|c|}
\hline Sl. No. & Particulars & Number & Per cent \\
\hline $\mathbf{1 .}$ & Indoor exhibition & 249 & 49.80 \\
\hline $\mathbf{2 .}$ & Consultancy cell & 81 & 16.20 \\
\hline $\mathbf{3 .}$ & Demonstrations & 58 & 11.60 \\
\hline $\mathbf{4 .}$ & Farmer -farmer interaction & 58 & 11.60 \\
\hline $\mathbf{5 .}$ & Field visits & 46 & 9.20 \\
\hline $\mathbf{6 .}$ & Stalls & 43 & 8.60 \\
\hline $\mathbf{7 .}$ & Farmer award function & 34 & 6.80 \\
\hline $\mathbf{8 .}$ & Input availability & 29 & 5.80 \\
\hline $\mathbf{9 .}$ & Others & 9 & 1.80 \\
\hline
\end{tabular}


Table.18 Three best stalls as perceived by the respondents

\begin{tabular}{|c|c|c|c|}
\hline Sl. No. & Division/stall & Number & Per cent \\
\hline 1. & Floriculture & 93 & 18.60 \\
\hline 2. & Agriculture implements / machineries & 86 & 17.20 \\
\hline 3. & Vegetable division & 82 & 16.40 \\
\hline 4. & Fruit division & 81 & 16.20 \\
\hline 5. & Soil world & 46 & 9.20 \\
\hline 6. & Consultancy & 34 & 6.80 \\
\hline 7. & Livestock & 31 & 6.20 \\
\hline 8. & Outdoor Exhibition & 27 & 5.40 \\
\hline 9. & Indoor exhibition & 16 & 3.20 \\
\hline 10. & Seed division & 12 & 2.40 \\
\hline 11. & Entomology & 10 & 2.00 \\
\hline 12. & Dog show & 8 & 1.60 \\
\hline 13. & Solar based machineries & 8 & 1.60 \\
\hline 14. & Food stalls & 8 & 1.60 \\
\hline 15. & Private institutes and NGO & 8 & 1.60 \\
\hline 16. & Post Harvest technology division & 5 & 1.00 \\
\hline 17. & Medicinal and aromatic division & 4 & 0.80 \\
\hline 18. & Social and allied sciences & 4 & 0.80 \\
\hline 19. & Banana stall & 4 & 0.80 \\
\hline 20. & Plant pathology division & 3 & 0.60 \\
\hline 21. & Publication & 3 & 0.60 \\
\hline 22. & Organic farming & 3 & 0.60 \\
\hline 23. & KVK, Kolar & 3 & 0.60 \\
\hline 24. & Rabbit rearing & 3 & 0.60 \\
\hline 25. & Research division & 1 & 0.20 \\
\hline 26. & Natural Resource Management & 1 & 0.20 \\
\hline 27. & Biofuel & 1 & 0.20 \\
\hline 28. & Sheep \& goat rearing & 1 & 0.20 \\
\hline 29. & UAHS, Shivmogga & 1 & 0.20 \\
\hline 30. & UAS, Bengaluru & 1 & 0.20 \\
\hline
\end{tabular}

Table.19 Number of respondents participated in farmer-farmer Interaction

\begin{tabular}{|c|l|c|c|}
\hline Sl. No. & \multicolumn{1}{|c|}{ Response } & Number & Per cent \\
\hline 1. & Participated & 143 & 28.60 \\
\hline 2. & Not participated & 357 & 71.40 \\
\hline
\end{tabular}


Table.20 Opinion of the respondents about farmer-farmer Interaction

\begin{tabular}{|c|l|c|c|}
\hline Sl. No. & \multicolumn{1}{|c|}{ Opinion } & Number & Per cent \\
\hline 1. & Best & 56 & 39.16 \\
\hline 2. & Better & 60 & 41.96 \\
\hline 3. & Useful & 26 & 18.18 \\
\hline 4. & Useless & 1 & 0.70 \\
\hline
\end{tabular}

Table.21 Opinion about the facilities provided to the respondents

\begin{tabular}{|c|c|c|c|c|c|c|c|c|c|}
\hline \multirow{2}{*}{$\begin{array}{c}\text { Sl. } \\
\text { No. }\end{array}$} & \multirow[t]{2}{*}{ Facilities } & \multicolumn{2}{|c|}{ Best } & \multicolumn{2}{|c|}{ Better } & \multicolumn{2}{|c|}{ Normal } & \multicolumn{2}{|c|}{ Poor } \\
\hline & & No & $\%$ & No & $\%$ & No & $\%$ & No & $\%$ \\
\hline 1 & Transport facility & 259 & 51.80 & 186 & 37.20 & 39 & 7.80 & 16 & 3.20 \\
\hline 2 & Interaction with scientists & 209 & 41.80 & 223 & 44.60 & 57 & 11.40 & 10 & 2.00 \\
\hline 3 & Publicity about the Mela & 251 & 50.20 & 207 & 41.40 & 33 & 6.60 & 9 & 1.80 \\
\hline 4 & Food arrangements & 145 & 29.00 & 240 & 48.00 & 93 & 18.60 & 22 & 4.40 \\
\hline 5 & Drinking $\mathrm{x}$ & 158 & 31.60 & 246 & 49.20 & 72 & 14.40 & 24 & 4.80 \\
\hline 6 & Parking facility & 230 & 46.00 & 229 & 45.80 & 35 & 7.00 & 6 & 1.20 \\
\hline 7 & Toilet facility & 160 & 32.00 & 266 & 53.20 & 65 & 13.00 & 9 & 1.80 \\
\hline 8 & Accommodation & 119 & 23.80 & 256 & 51.20 & 99 & 19.80 & 26 & 5.20 \\
\hline
\end{tabular}

Table.22 Useful and innovative technology/information as perceived by the respondents

\begin{tabular}{|c|l|c|c|}
\hline Sl. No. & \multicolumn{1}{|c|}{ Particulars } & Number & Per cent \\
\hline 1. & Technical information & 68 & 13.60 \\
\hline 2. & Machineries and implements & 46 & 9.20 \\
\hline 3. & Solar machineries & 25 & 5.00 \\
\hline 4. & Demonstration & 18 & 3.60 \\
\hline 5. & Livestock & 1 & 0.20 \\
\hline
\end{tabular}

Table.23 Opinion about adoption of technology received in Mela

\begin{tabular}{|c|l|c|r|}
\hline Sl. No. & Opinion & Number & Per cent \\
\hline 1. & Adopt & 89 & 56.32 \\
\hline 2. & Will not adopt/ not decided & 69 & 43.68 \\
\hline
\end{tabular}

Table.24 Overall perspective of the respondents about TotagarikaMela

\begin{tabular}{|c|l|c|c|}
\hline Sl. No. & Opinion & Number & Per cent \\
\hline 1. & Best & 246 & 49.20 \\
\hline 2. & Better & 191 & 38.20 \\
\hline 3. & Normal & 62 & 12.40 \\
\hline 4. & Poor & 1 & 0.20 \\
\hline
\end{tabular}


Table.25 Suggestions for the improvement of Totagarike Mela

\begin{tabular}{|c|l|c|c|}
\hline SI. No. & Suggestions & Number & Per cent \\
\hline $\mathbf{1 .}$ & Improvement in Food arrangements & 38 & 7.60 \\
\hline $\mathbf{2 .}$ & Improvement in Drinking Water facility & 21 & 4.20 \\
\hline $\mathbf{3 .}$ & Consultancy & 21 & 4.20 \\
\hline $\mathbf{4 .}$ & Free and more transportation facility & 18 & 3.60 \\
\hline $\mathbf{5 .}$ & More publicity & 18 & 3.60 \\
\hline $\mathbf{6 .}$ & Improvement and increase in demonstration plots & 13 & 2.60 \\
\hline $\mathbf{7 .}$ & Free and good Accommodation facility & 9 & 1.80 \\
\hline $\mathbf{8 .}$ & Cleanliness & 4 & 0.80 \\
\hline $\mathbf{9 .}$ & Toilet facility & 4 & 0.80 \\
\hline $\mathbf{1 0}$. & Early publicity of experts and progressive farmers participants & 1 & 0.20 \\
\hline $\mathbf{1 1}$. & Importance to livestock exhibition & 1 & 0.20 \\
\hline $\mathbf{1 2}$. & Increase in post harvest technology stalls & 1 & 0.20 \\
\hline $\mathbf{1 3}$ & More Seedlings availability & 1 & 0.20 \\
\hline
\end{tabular}

Table.26 Motivation of Respondents to others to participate in Mela

\begin{tabular}{|r|l|c|c|}
\hline Sl. No. & Particular & Numbers & Per cent \\
\hline 1. & Motivated & 236 & 47.20 \\
\hline 2. & Not motivated & 274 & 54.80 \\
\hline
\end{tabular}

Opinion about adoption of technology received in Mela

Out of 158respondents who received useful and innovative technology/information as perceived by them, only 56.32per cent will adopt while remaining 43.68per cent were not decided to adopt or may not adopt (Table 23).

Overall perspective of the respondents about Totagarika Mela

We learnt from the table 24 that nearly half of the respondents $(49.20 \%)$ opined that the totagarike mela was best followed by better $(38.20 \%)$ and normal $(12.40 \%)$. While only one person said the mela was poor which might be due to his higher expectations.
Suggestions for the improvement of Totagarike Mela

In order to improve the Totagarike Mela in future, Respondent participants suggested to improve food arrangements $(7.60 \%)$, drinking water facility and consultancy $(4.20 \%$ each), free and more transport facility and more publicity $(3.60 \%$ each), improvement and increase in demonstration plots $(2.60 \%)$, free and good accommodation facility (1.80\%). While less than one per cent of the respondents suggested to improve cleanliness, toilet facility, early publicity of experts and progressive farmers participants, livestock exhibition, increase post harvest technology display stalls and more seedlings availability (Table 25). 


\section{Number of Respondents motivated others} to participate in Mela

About 47.20per cent of the respondent participants motivated friends, neighbours, relatives, etc to participate in the Mela while remaining didn't motivate any other (Table 26).

It could be concluded from this study that majority of respondent were from Bagalkot district, middle aged, illiterates with small land holding and agriculture was their major occupation. Majority of them obtained information about mela from newspaper followed by TV, line departments and friends and other sources. Good majority of farmers perceived that, new information was received agriculture machineries, soil health management, horticulture crops, etc.

Among different divisions, most of the participants rated floriculture, machineries, vegetable and fruit division as the best where as majority of them didn't participated in farmer to farmer interaction which needs attention. Overall half of the respondents opined the totagarike mela as the best. The major suggestions expressed by the respondents were improvement in food and drinking water facility for future.

\section{References}

Gangadharappa, N.R. and Jayaramaiah, K.M., 1985, A critical analysis of Krishimela. Curr. Res., 14 (1-3): 19:20.

Manjula, N. and M. K. Sheikh, 2010, Impact of Krishimela on participating farmers Agriculture Update, Vol. 5 (1\&2) 5458.\& 2| 54-58 |Agriculture Update | February \& May 2010 | Vol. 5 MM | Issue $1 \& 2 \mid$ 54-58.

Manjula, N., Gowda, G.V., Shashikumar, S. and Kumari Roopa G., 2002, Perception of farm women about KrishiMela held atUniversity of Agriculture Sciences, Bangalore. Land Bank J., Pp. 13-19.

Narayanaswamy, B., Ramakrishna, Naika, Nataraj, M.S. and Narayanagowda K. 2005, Opinion of farmers on Krishimela and their suggestions.

Rao, M.K., Venkataramaiah, P. and Hanchinal, S.N. 1996, An appraisal of field day programme 1974-75 by the participants conducted at College of Agriculture, Dharwad, Curr. Res., 5: 24

Swamy, S.B., Jahagirdar, K. A. and Sontakki, B.S. 1992, Characteristics of participants of Krishimela, Maharastra J.Extn. Edu., 11: 319-323.

\section{How to cite this article:}

Lakshmana Reddy, B.S., Pushpa and Srinivas Reddy, M.V. 2020. Impact Assessment of Horticulture Fair on Farming Community. Int.J.Curr.Microbiol.App.Sci. 9(08): 1282-1296. doi: https://doi.org/10.20546/ijcmas.2020.908.145 